
\title{
25 Research Soure \\ Sex Differences in the Relationship of Hip Strength and Functional Performance to Chronic Ankle Instability Scores
}

Junlan Lu

Tongji Hospital of Tongji Medical College of Huazhong University of Science and Technology

\section{Zhigang Wu}

Hainan western central hospital

Roger Adams

University of Canberra

Jia Han

Shanghai University of Sport

Cai Bin ( $\nabla$ shrehab@163.com )

Hainan western central hospital https://orcid.org/0000-0001-8634-0580

\section{Research Article}

Keywords: ankle, hip, sex, functional performance, rehabilitation

Posted Date: December 28th, 2021

DOI: https://doi.org/10.21203/rs.3.rs-1190199/v1

License: (c) (i) This work is licensed under a Creative Commons Attribution 4.0 International License. Read Full License 


\section{Abstract}

Background: Preliminary studies have reported differences in strength and functional performance between sexes for patients with anterior cruciate ligament injury or reconstruction. Similar sex differences may occur in chronic ankle instability (CAI) populations. Factors like hip abduction strength and functional performance may be contributors to sex differences in CAI populations, but their presence and magnitude is unclear. While decreased hip abductor strength, functional performance, and self-reported instability scores have all been shown in association with CAl, any sex difference in the relationship between these indicators is unclear. The study was to determine if sex differences are present in the relationship between these indicators in individuals with CAI.

Methods $\triangle T$ Thirty-two women and twenty-nine men with unilateral CAI took part. Hip abductor strength and functional performance were respectively assessed using a hand-held dynamometer and the figure-8-hop test. All 61 participants scored the Cumberland Ankle Instability Tool (CAIT) for self-reported ankle instability.

Results: Normalized hip abductor strength and functional performance measures for females were lower than for males. The self-reported ankle instability CAIT score, where higher values represent less instability, was significantly and positively correlated with normalized hip abductor strength and functional performance on the affected side in females (all $p<0.01$ ), but not in males (all $p>0.19$ ).

Conclusions: Sex differences were observed in the relationships of normalized hip abductor strength and functional performance to CAIT scores from the CAl, holding for females but not males, and suggesting that evaluation and rehabilitation strategies should be sex-specific.

\section{Highlights}

1. In females with CAl, hip abductor strength and functional performance showed significant relationships with self-reported instability scores.

2. Correspondingly, in clinical practice, evaluation criteria may be formulated according to these observed sex differences in individuals with CAl.

3. Sex differences should be factored into the evaluation and treatment of CAI individuals.

4. Hip strength assessment should be specific in CAI individuals.

5. Hip strengthening and functional hopping may be recommended for the rehabilitation of CAIQ especially in female patients.

\section{Background}


Ankle sprain is the most common sports injury.[1] Approximately $40 \%$ of patients with ankle sprains experience repeated sprains, "giving way," and other symptoms associated with chronic ankle instability (CAl).[2] Females were found to be more likely to report sprains and to suffer CAl than males. $[3,4]$

CAl populations are defined by their lower self-reported ankle function across a variety of tasks, and they frequently display deficits in functional performance. The Cumberland Ankle Instability Tool (CAIT) is one way of quantifying CAI. It can reliably and validly evaluate the subjective response of individuals regarding ankle performance,[5] including self-evaluation of pain, sense of stability and "giving way" in daily life and during physical activities. Those classified with CAI have lower self-reported scores than people with healthy ankles, indicating that they have more functional limitations.[6]

In addition to symptom scores, CAl individuals also have decreased functional performance. The figure-8hop test is a functional task that requires speed, power, and agility, and it challenges the stability of the lower limb joints in individuals with CAI. The test provides high clinical utility, and is widely-used with CAI populations. In their results, Docherty et al.[7] found a positive relationship between CAIT scores and figure-8-hop test performance. However, most of the participants (71.6\%) in their study were female. Given that the incidence of acute ankle sprains and CAI in female athletes is approximately twice as high as that in male athletes, $[3,4]$ the CAIT-performance relationship may be different between males and females. Further, functional task performance may be affected by hip strength deficits.[8] Hip abductor strength is significantly diminished in CAI individuals $[8,9]$ and other studies have reported sex differences of hip strength in patients with patellofemoral pain or anterior cruciate ligament injury.[10, 11] It can be proposed that similar sex differences might be found in a CAI population. However, sex differences in the relationships between these variables have not previously been examined, although this would be relevant to current understanding of sex differences in functional impairments in CAl, and inform regarding possible need for sex-specific evaluation and rehabilitation.

This study aimed to explore sex differences in the relationships between hip abductor strength, functional performance, and self-reported instability scores in CAI individuals. It was hypothesized that hip abductor strength and functional performance are positively related to self-reported instability scores, but may that this relationship may differ between males and females. Given the incidence of acute ankle sprains and CAl found in females, it could also be hypothesized that stronger relationships between hip strength, functional performance, and self-report CAIT scores would be found in females compared with males. The significance here is that if the relationship between male and female individuals with CAI is distinctly different, evaluation criteria may need to be adjusted and targeted interventions designed accounting for any sex differences.

\section{Methods}

Sixty-one participants, of both sexes and aged between 18 and 45 years, were recruited through advertisements. The female group consisted of 32 participants, and the male group consisted of 29 participants, using a diagnosis of unilateral CAI made by following the International Ankle Consortium's 
criteria for CAl from Gribble et al.[12] Inclusion criteria[12] were: 1) at least one severe ankle sprain, leading to a day off, 2) the first sprain occurred at least 1 year ago, with no sprain in the last 3 months, 3) experiencing "giving way" or repeated sprains, and 4) a CAIT score for the affected limb that was $\leq 24$. Exclusion criteria were: 1) history of lower extremity surgery, 2) lower extremity fracture, 3) acute musculoskeletal injury of the lower extremity affecting joint integrity and function within the 3 months before the current study, with at least 1 day of physical activity disability, 4) a previous ankle sprain on the "non-affected" limb, 5) CAIT score of the contralateral limb $₫ 28$. Characteristics of the participants are provided in Table 1. The study was approved by the relevant institution's human ethics committee (Approval number: SH9H-2019-T54-2), and written informed consent was obtained from all participants before data collection commenced.

Table 1

Baseline demographics of participants $(\mathrm{N}=61$; mean $\pm \mathrm{SD})$

\begin{tabular}{|llll|}
\hline Participant characteristics & Male $(\mathbf{n}=29)$ & Female $(\mathrm{n}=\mathbf{3 2})$ & $\boldsymbol{p}$ \\
\hline Age, $\mathrm{y}$ & $34.9 \pm 5.2$ & $30.0 \pm 5.6$ & 0.001 \\
\hline Height, $\mathrm{cm}$ & $175.2 \pm 4.5$ & $163.9 \pm 5.2$ & 0.000 \\
\hline Weight, $\mathrm{kg}$ & $72.0 \pm 7.2$ & $57.3 \pm 7.2$ & 0.000 \\
\hline BMl, $\mathrm{kg} / \mathrm{m}^{2}$ & $23.4 \pm 2.1$ & $21.3 \pm 2.5$ & 0.001 \\
\hline Hip Abductor Strength, Nm/kg & & & \\
\hline Affected side & $2.1 \pm 0.5$ & $1.7 \pm 0.4$ & 0.000 \\
\hline Figure-of-8 Hop Test, s & & & 0.005 \\
\hline Affected side & $6.8 \pm 2.2$ & $8.2 \pm 1.6$ & \\
\hline Cumberland Ankle Instability Tool, score & & & 0.222 \\
\hline Affected side & $14.6 \pm 5.6$ & $12.7 \pm 6.3$ & \\
\hline
\end{tabular}

Upon enrolling in the study, participants first completed the Cumberland Ankle Instability Tool, evaluating the stability of both ankle joints. Participants were blinded to the study hypothesis. Then, hip abductor strength and functional performance were measured and documented by an experienced physiotherapist who was blinded to the study hypothesis, and the CAIT score of each participant. The order of testing of hip abductor strength and functional performance was randomized. A statistician who was blinded to the study hypothesis performed the data analysis.

Participants were asked to complete the CAIT to determine the severity of their subjective symptoms associated with ankle instability. The CAIT [5] is a nine-item questionnaire intended to identify and grade ankle instability. It includes questions addressing activities that cause pain or instability, how often individuals experience an ankle sprain, and how quickly they recover from those episodes. Each answer is assigned a value ranging from 0 to 5 . The instrument has excellent test-retest reliability, with an 
intraclass correlation coefficient of 0.96.[5] Participants can receive a maximum score of 30, indicating no symptoms, with a score lower than 24 suggesting CAl.

A MicroFET2 hand-held dynamometer (Hoggan Industries, Inc., West Jordan, UT, USA) was used to measure maximal voluntary isometric contraction of the hip abductors. The device has a battery-operated load-cell system that provides a digital reading of peak force, expressed in Newtons, and was calibrated before testing. Femur length was measured by a tape from the femoral trochanter to lateral knee joint line, for normalization of isometric hip strength outcomes. Test-retest reliability for this measure of hip abductor strength is good (Intraclass correlation coefficient $=0.76$ ).[13] During measurement of hip abductor strength, participants were in a side-lying position with the iliac crest stabilized.[14] The dynamometer was placed $5.08 \mathrm{~cm}$ proximal to the knee joint line to implement the "make-test". [14]

One practice trial was followed by three tests. Standardized instructions were given where participants increased contraction intensity for the first three seconds, then provided maximal effort for the fourth and fifth seconds. The maximum force measured was used as the participant's peak force. Because participants differed in femur length and body weight, the standardized final outcome measure of hip abductor strength was calculated using the following equation: [14]

\section{Normalized hip abductor strength $(\mathrm{Nm} / \mathrm{kg})=$ Peak force * Femur length/weight}

The figure-of-8 hop test was used as a functional performance measure for individuals with CAI. Results from this test have been positively correlated with answers to questions on self-reported feelings of ankle instability, indicating that greater feeling of instability is related to increased time taken to complete the test.[7] Test reliability has been found to be excellent, with an intraclass correlation coefficient of 0.95.[15] In the present study, participants performed the test barefoot on a 5-m course outlined by cones in a figure-of-8 pattern (Figure 1). Each participant was instructed to hop as fast as possible, on one leg, through the course twice. Time to complete the task was recorded with a stopwatch, to the nearest 0.1 second. Participants could perform an additional trial if they failed to complete the course. ${ }^{7}$ Participants were tested twice, and the shorter time used for analysis.[7]

The Kolmogorov-Smirnov test was used to examine normality of the data distribution. Demographic characteristics of both female and male groups are presented in Table 1, and independent sample t-tests were used to examine between group differences. Separate Spearman's correlations were conducted between normalized hip abductor strength and CAIT, and functional performance and CAIT, within each group. The relationship between hip abductor strength and functional performance was examined by Pearson's product moment correlation coefficients within each group separately. Correlation coefficients of 0 to $0.3,0.3$ to 0.5 , and 0.5 to 1.0 represented a weak, moderate, or strong correlation, respectively.[16] All statistical analyses were performed using SPSS software (version 22.0; IBM Corporation, Armonk, NY, USA). 


\section{Results}

The age, height, weight, and BMI of the female group were significantly lower than those of the male group (Table 1). Female and male CAI individuals reported a similar severity of ankle instability based on their CAIT score on the affected side $(p>0.05)$. Normalized hip abductor strength for male participants was significantly greater than that of female participants on affected sides $(p<0.05)$. Male participants performed significantly better than their female counterparts in the figure-of-8 hop test for the affected side $(p<0.05)$.

Clear differences were observed in the correlation measures between female and male CAI individuals. In females with CAl, self-reported ankle instability scores were significantly correlated with normalized hip abductor strength on the affected side ( $r h o=0.52, p<0.01)$, indicating a moderate positive correlation (Table 2). The CAIT score was significantly also correlated with functional performance on the affected side ( $r h o=-0.57, p<0.01)$ in female participants with CAl, indicating a moderate negative correlation (Table 2). However, in males with CAl, no significant correlations were observed between normalized hip abductor strength or functional performance and self-reported ankle instability scores on the affected side (all $p>0.05$ ).

Table 2

Relationship of hip abductor strength and functional performance with CAIT scores in individuals with CAI

\begin{tabular}{|lllll|}
\hline Measurements & \multicolumn{2}{c|}{ Male $(\mathrm{n}=29)$} & \multicolumn{2}{l|}{ Female $(\mathbf{n}=32)$} \\
\cline { 2 - 5 } & \multicolumn{2}{c|}{ Affected side } & \multicolumn{2}{c|}{ Affected side } \\
\cline { 2 - 5 } & rhos & $\boldsymbol{p}$ & hos & $\boldsymbol{p}$ \\
\hline Hip Abductor Strength & 0.18 & 0.361 & 0.52 & $0.003^{\star}$ \\
\hline Figure-of-8 Hop Test & -0.25 & 0.192 & -0.58 & $0.001^{\star}$ \\
\hline
\end{tabular}

In this cohort of individuals with CAI, normalized hip abductor strength was moderately and significantly correlated with functional performance on the affected side with $r$ values that ranged from -0.44 to -0.37 (all $p<0.05$ ).

\section{Discussion}

This study set out to explore the association between hip abductor strength, functional performance, and ankle instability (CAIT) scores in males and females with CAI. A statistically significant correlation between hip abductor strength, functional performance, and CAIT scores was observed in female, but not in male, participants, an outcome consistent with our initial hypothesis. 
This finding may be related to sex differences in anatomical structure and postural control patterns. The quadriceps angle ( $Q$ angle) is an important indicator of biomechanical function and normal alignment of the lower leg, providing useful information underpinning the functional ability of the lower extremity. The $\mathrm{Q}$ angle of women is approximately $5.8^{\circ}$ larger than that of men. With greater Q-angles, women are therefore believed to be at greater risk of patellofemoral pain than men. [16] It may also be a factor in the presence of CAl. Larger $\mathrm{Q}$ angles increase the demand for hip abductor muscle activation during landing. [11] In theory, greater hip muscle activation would be necessary to successfully perform a desired motion in the presence of reduced hip muscle strength. After ankle sprain, hip abductor strength here was affected compared to controls, so it is less surprising that female individuals with CAI have worse CAIT scores. In addition, studies have shown that the pattern of postural stability control differs by sex in heathy populations.[17] Women are more likely to use an ankle strategy to resist external interference than men. [18] When an ankle sprain occurs, ankle proprioception is impaired,[19] and use of ankle strategies may be compromised in the CAI population. According to the concept of a lower limb movement chain, the hip strategy of women may be oriented towards compensating, but compensation at the hip requires high hip abductor strength. In addition, hip abductor muscle strength plays an important role in maintaining balance and stability in the frontal plane.[20] Further, females are more likely to report symptoms and seek medical care across all injuries. Therefore, the weaker hip abductor muscle strength observed in female participants in the current study may contributed to the worse CAIT sores obtained in this group.

Compared to males, women's functional performance was also more strongly correlated with CAIT scores. This may be related to sex differences in one-leg jump landing strategy and fear of ankle re-sprain .$[21,22]$ Previous studies have shown that in a functional task like single-leg jump landing, females showed a more rigid landing strategy, and female' knee joints absorbed $4.5 \%$ more energy than male knee joints, whereas male hip joints absorbed $4.3 \%$ more energy than female hip joints. [21] Additionally, females with limited sagittal plane motion during landing exhibit a biomechanical profile that may put them at greater risk for anterior cruciate ligament injury. [23] The female energy absorption strategy and sagittal plane motion may reflect their hip muscle weakness, [21,23] such that females with weak hip strength may have poorer performance and have more injury risk in the functional task than males. The functional task used in this study was the figure-8-hop test, which includes multiple single-leg jump landing movements. The CAIT self-reported instability scale involves the evaluation of one-foot jump and other movements, and for those with CAl, perception of instability may be more acute, which may result in the observed worse CAIT scores of female CAl individuals. Therefore, to complete a functional task well, such as the one employed here, requires adequate hip abductor strength, especially in females. Moreover, researchers have reported that fear of movement increased after an ankle sprain, as observed from responses when filling out the Fear-Avoidance Belief Questionnaire (FABQ).[24] A moderate correlation has been observed between the CAIT score and the FABQ score. [25] Ankle instability is positively correlated with fear. ${ }^{25}$ Comparing the ankle instability for individuals of different sexes, results revealed that the ankle instability of female patients was related to fear.[22] Studies have shown that constant fear 
of injury is a major obstacle to exercise and activity. [26] Therefore, women with ankle sprains be more likely to have fear of re-sprain, which may affect their CAIT scores.

The significance of this study is that in exploration of the correlation between normalized hip abductor strength, functional performance and CAIT scores in male and female patients with CAI, different correlations were revealed in CAI individuals of different sexes. Specifically, a statistically significant correlation was found for female normalized hip abductor strength, functional performance, and CAIT score. Thus, in future rehabilitation evaluation, corresponding evaluation criteria should be formulated according to sex differences. Moreover, female patients should get more attention regularly in clinical settings, especially in terms of their hip abductor strength, and targeted interventions should be implemented to solve the problem.

This study has some limitations. First, the time after ankle sprain differed among participants. After a longer time, ankle injury may have a chronic psychological impact, affecting self-reported instability. [27] The CAIT scores of the patients with less than 2 times of sprain frequency was significantly higher than that of the patients with a higher frequency of sprain. In the future, the course of response in patients with ankle sprains should be taken into account. Second, the incidence of ankle sprains was different in different age groups. [28] Physical activity level and choice of activity in different age or different sex may be relevant to the study. The female participants included in the present study were significantly younger and no assessment of possible sex differences in these relationships was performed among CAI individuals of different age groups.

One previous study showed that normalized hip abductor strength training could effectively improve neuromuscular control, strength, and self-reported function in individuals with CAI, [29] and a recent review has suggested that adequate normalized hip abductor strength is important in the rehabilitation of CAl individuals, [30] so hip abductor strength training is implicated here.

\section{Conclusion}

In females but not in males with CAl, this study found statistically significant relationships between normalized hip abductor strength, functional performance and self-reported instability scores. In the future, CAl evaluation criteria should be formulated according to sex differences, and attention paid to hip strengthening and functional hopping in CAI rehabilitation with female patients.

\section{Abbreviations}

CAl: chronic ankle instability; CAIT: Cumberland Ankle Instability Tool.

\section{Declarations}

\section{Acknowledgements}


None.

\section{Authors' contributions}

$\mathrm{JL}$ and ZW contributed to the conceptualization, formal analysis, methodology, project administration, visualization, and writing-original draft. JL, $\mathrm{ZW}, \mathrm{BC}$ and $\mathrm{JH}$ contributed to the data curation, visualization, writing-review and editing. $\mathrm{RA}, \mathrm{BC}$ and $\mathrm{JH}$ contributed to the conceptualization, supervision, and writing-review and editing. All authors read and approved the final manuscript.

\section{Funding}

This work was supported by the Scientific research project of Hainan Health Committee [grant number 20A200490].

\section{Availability of data and materials}

The datasets used and/or analyzed during the current study are available from the corresponding author on reasonable request.

\section{Ethics approval and consent to participate}

The study was approved by the Shanghai Ninth People's Hospital, Shanghai Jiao Tong University School of Medicine Institutional Review Board (SH9H-2019-T54-2). Each subject provided informed written consent form at the time of recruitment.

\section{Consent for publication}

Not applicable.

\section{Competing interests}

The authors declare no potential conflicts of interest. All procedures in this study involving human participants were in accordance with the ethical standards of the institutional research committee and with the 1964 Helsinki Declaration and its later amendments or comparable ethical standards.

The authors report no proprietary or commercial interest in any product mentioned or concept discussed in this article. 


\section{References}

1. Fong TP, Hong Y, Chan LK. A systematic review on ankle injury and ankle sprain in sports. Sports Med. 2007;37(1):73-94.

2. Hershkovich 0 . A large-scale study on epidemiology and risk factors for chronic ankle instability in young adults. J Foot Ankle Surg. 2015, 54(2):183-187.

3. Tanen L, Docherty CL, Van DP. Prevalence of chronic ankle instability in high school and division I athletes. Foot Ankle Special. 2014, 7(1):37-44.

4. Doherty $C$, Delahunt E, Caulfield B. The incidence and prevalence of ankle sprain injury: a systematic review and meta-analysis of prospective epidemiological studies. Sports Med. 2014;44(1):123-40.

5. Hiller CE, Refshauge KM, Bundy AC. The cumberland ankle instability tool: a report of validity and reliability testing. Arch Phys Med Rehab. 2006;87(9):1235-41.

6. Houston MN, Hoch JM, Hoch MC. Patient-reported outcome measures in individuals with chronic ankle instability: a systematic review. J Athl Train. 2015, 50(10):1019-1033.

7. Docherty $\mathrm{CL}$, Arnold BL, Gansneder BM. Functional-performance deficits in volunteers with functional ankle instability. J Athl Train. 2005, 40(1):30-34.

8. Mccann RS, Terada M, Kosik KB. Landing kinematics and isometric hip strength of individuals with chronic ankle instability. Foot Ankle Int. 2019, 40(8):969-977.

9. Kosik KB, Johnson NF, Terada M. Decreased ankle and hip isometric peak torque in young and middle-aged adults with chronic ankle instability. Phys Ther Sport. 2020, 43(12):345-349.

10. Nakagawa T, Moriya E, Maciel C. Trunk, pelvis, hip, and knee kinematics, hip strength, and gluteal muscle activation during a single-leg squat in males and females with and without patellofemoral pain syndrome. J Orthop Sports Phys Ther. 2012, 42(6):491-501.

11. Nguyen A, Shultz S, Schmitz R. A preliminary multifactorial approach describing the relationships among lower extremity alignment, hip muscle activation, and lower extremity joint excursion. $J$ Athl Train. 2011, 46(3):246-256.

12. Gribble P, Delahunt E, Bleakley C. Selection criteria for patients with chronic ankle instability in controlled research: a position statement of the International Ankle Consortium. Br J Sports Med. 2014, 48(13):1014-1018.

13. Thorborg K, Petersen J, Magnusson S. Clinical assessment of hip strength using a hand-held dynamometer is reliable. Scand J Med Sci Sports. 2010, 20(3):493-501.

14. Mccann RS, Crossett ID, Terada M. Hip strength and star excursion balance test deficits of patients with chronic ankle instability. J Sci Med Sport. 2017, 20(11):992-996.

15. Caffrey E, Docherty CL, Schrader J. The ability of 4 single-limb hopping tests to detect functional performance deficits in individuals with functional ankle instability. J Orthop Sport Phys. 2009, 39(11):799-806.

16. Caylor D, Fites R, Worrell TW. The relationship between quadriceps angle and anterior knee pain syndrome. J Orthop Sports Phys Ther. 1993;17(1):11-16. 
17. Chen Z, Han J, Waddington G. Somatosensory perception sensitivity in voluntary postural sway movements: age, gender and sway effect magnitudes. Exp Gerontol. 2019;122(3):53-59.

18. Olchowik G, Tomaszewski M, Olejarz P. The human balance system and gender. Acta Bioeng Biomech. 2015, 17(1):69-74.

19. Witchalls J, Waddington $G$, Blanch P. Ankle instability effects on joint position sense when stepping across the active movement extent discrimination apparatus. J Athl Train. 2012, 47(6):627-634.

20. Ambegaonkar JP, Mettinger LM, Caswell SV. Relationships between core endurance, hip strength, and balance in collegiate female athletes. Int J Sports Phys Ther. 2014, 9(5):604-616.

21. Montgomery MM, Shultz SJ, Schmitz RJ. The effect of equalizing landing task demands on sex differences in lower extremity energy absorption. Clin Biomech (Bristol, Avon). 2014, 29(7):760-766.

22. Fukano M, Mineta S, Hirose N. Fear avoidance beliefs in college athletes with a history of ankle sprain. Int J Sports Med. 2020, 41(2):128-133.

23. Pollard C, Sigward S, Powers C. Limited hip and knee flexion during landing is associated with increased frontal plane knee motion and moments. Clin Biomech (Bristol, Avon). 2010, 25(2):142146.

24. Houston M, Hoch J, Hoch M. College athletes with ankle sprain history exhibit greater fear-avoidance beliefs. J Sport Rehabil. 2018, 27(5):419-423.

25. Mohamadi S, Dadgoo M, Ebrahimi I. Translation, cross-cultural adaptation, reliability, and validity of the identification of functional ankle instability questionnaire in persian speaking participants with a history of ankle sprain. Disabil Rehabil. 2019, 41(16):1931-1936.

26. Lysdal F, Bandholm T, Tolstrup J. Does the spraino low-friction shoe patch prevent lateral ankle sprain injury in indoor sports? a pilot randomised controlled trial with 510 participants with previous ankle injuries. Br J Sports Med. 2020, 17(3):449-454.

27. Jaber H, Lohman E, Daher N. Neuromuscular control of ankle and hip during performance of the star excursion balance test in subjects with and without chronic ankle instability. PLoS One. 2018, 13(8):-1471-1479.

28. Waterman BR, Owens BD, Davey S. The epidemiology of ankle sprains in the United States. J Bone Joint Surg Am. 2010, 92(13):2279-2284.

29. Smith BI, Docherty CL, Curtis D. Effects of hip strengthening on neuromuscular control, hip strength, and self-reported functional deficits in individuals with chronic ankle instability. J Sport Rehabil. 2017, 27(4):364-370.

30. Khalaj N, Vicenzino B, Heales LJ. Is chronic ankle instability associated with impaired muscle strength? Ankle, knee and hip muscle strength in individuals with chronic ankle instability: a systematic review with meta-analysis. Br J Sports Med. 2020, 54(14): 839-847.

\section{Figures}




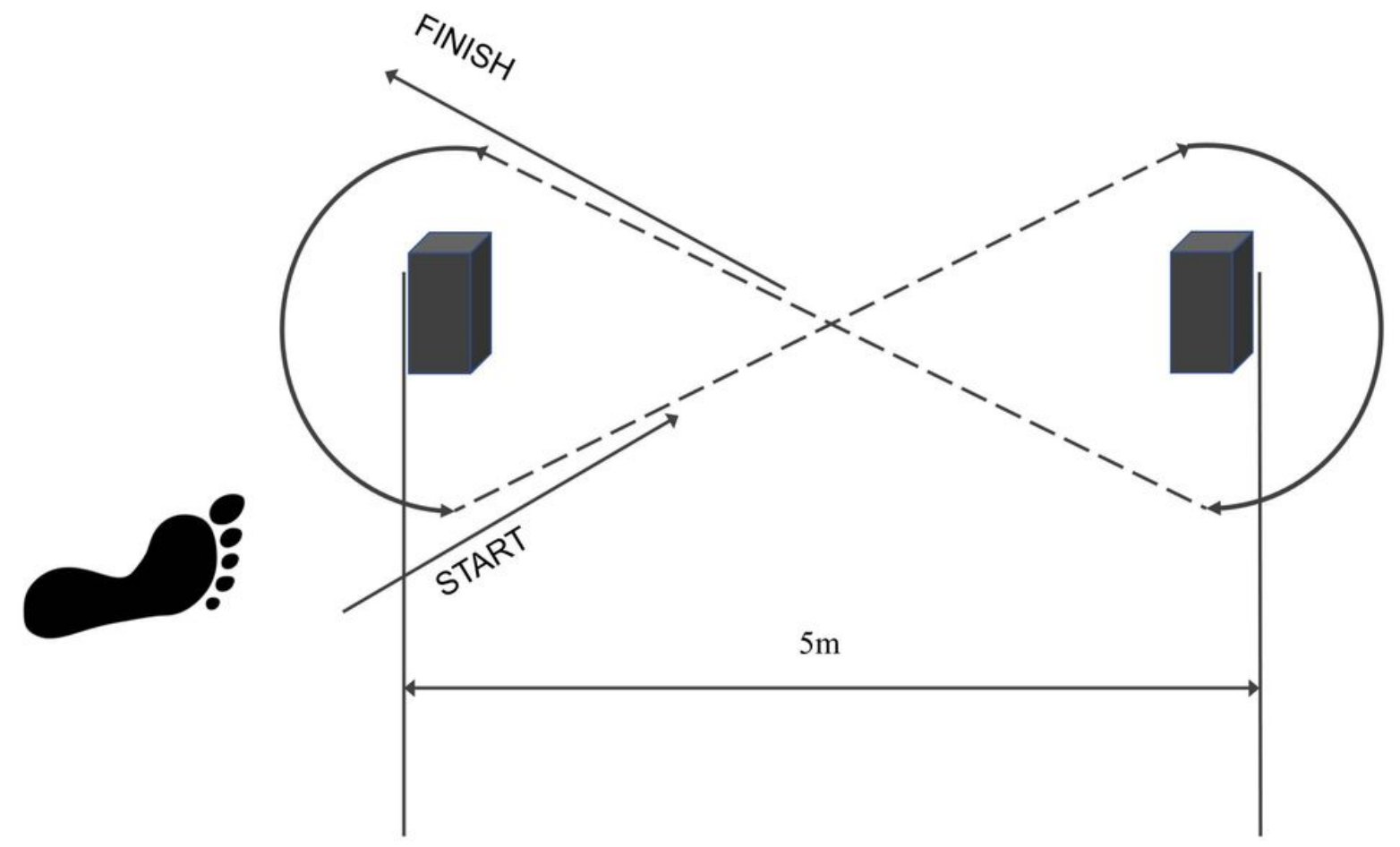

Figure 1

Figure-of-8 hop test 